\title{
STUDY ON FINANCIAL ANALYSIS OF ICICI BANK
}

\author{
K. Neelavathi \\ Lecturer in Commerce, \\ Bhavan's Vivekananda College of Science, Humanities, and Commerce, \\ Hyderabad, India \\ Ramya Chavali \\ Student, Institute of Public Enterprise, \\ Business School in Secunderabad, Telangana, India

\section{Rohan Bhavirisetty} \\ Student, Bhavan's Vivekananda College of Science, Humanities, and Commerce, \\ Hyderabad, India
}

\begin{abstract}
The Indian banking market is growing at an astonishing rate. The banks are being segregated into different groups. Each group has its benefits and limitations in operating in India. ICICI Bank is India's largest private sector bank. The bank's consolidated total assets stood at Rs. 12.50 trillion on June 30 2019. ICICI Bank currently features a network of 5,275 branches and 15,589 ATMs across India. The analysis is formed by considering the financial statements of the past five years. The income of the bank has increased over the amount. The bank has succeeded in maintaining an inexpensive profitability position. The bank continued to expand its branch network in India. The Research Accelerates towards assessing ICICI Bank's profitability to work out the Financial situation of ICICI Bank. It ends at measuring the liquidity position and analyses ICICI Bank's solvency, which provides us a crystal-clear insight into the financial Journey of the BANK.
\end{abstract}

Key Words: ICICI BANK, Financial Analysis, Comparative Financial Statements, Ratio Analysis and, Common Size Statements

Cite this Article: K. Neelavathi, Ramya Chavali and Rohan Bhavirisetty, Study on Financial Analysis of ICICI Bank, International Journal of Management, 11(12), 2020, pp 3388-3402.

http://www.iaeme.com/IJM/issues.asp?JType=IJM\&VType=11\&IType=12 


\section{OBJECTIVES OF THE STUDY}

- To assess the profitability of ICICI Bank.

- To determine the financial position of ICICI Bank.

- To measure the liquidity position and analyze the solvency of ICICI Bank

\section{INTRODUCTION}

\subsection{Company Profile}

\section{Introduction to ICICI Bank}

ICICI Bank is India's largest private sector bank with a full range of combined Bank assets. 12.50 trillion as of June 30 2019. ICICI Bank currently has a network of 5,275 branches and 15,589 ATMs across India.

Its origin in the simplest form can be traced to the source of authentic history. After recognizing the benefit of money as a medium of exchange, the importance of banking was developed as it provides a safer place to store money. This safe place ultimately evolved into financial institutions that accept deposits and make loans, i.e., modern Commercial banks.

ICICI (Industrial Credit and Investment Corporation of India) Bank was first established in 1994 by ICICI Limited, India's financial institution, and was its wholly-owned subsidiary. The ICICI Bank's shareholding of ICICI Bank has been reduced by $46 \%$ with the public offering of shares in India in the 1998 financial year, contributing to the ADR system listed on the NYSE in the 2000 financial year, the acquisition of ICICI Bank by Madura Limited.

In the consolidation of total stock for the 2001 financial year and the second market sale by ICICI to institutional investors for the 2001 financial year and the 2002 financial year. ICICI was established in 1955 at the World Bank's initiative, the Government of India, and representatives of the Indian industry. The main objective was to establish a financial center to develop funding for medium to long-term projects in Indian businesses.

In the 1990s, ICICI transformed its business from a development finance development center that provides only project funding to a diverse financial services provider that offers a wide range of products and services, both directly to corporate and organizations such as ICICI Bank. In 1999, ICICI became the first Indian company with the first bank or financial institution from a non-Japan Asia to be listed on the NYSE.

After considering various forms of corporate governance in the context of the competitive environment in the Indian banking industry and general banking, the executives of ICICI and ICICI Bank formulate the idea that merging ICICI with ICICI Bank will be an excellent strategy for both parties and will create a better legal framework for ICICI group banking. The merger will improve the number of ICICI shareholders through integrated business access to low-cost deposits, critical earnings, and the ability to participate in the payment system and provide transaction banking services. The merger will improve the number of ICICI Bank shareholders through an extensive financial base and operating rate, seamless access to ICICI strong relationships built over 50 years, entry into new business units, high market share in various business categories, especially billing services, and access to a large pool of the talents of ICICI and its subsidiaries.

In October 2001, the Board of Directors of ICICI and ICICI Bank approved ICICI and two wholly-owned commercial companies, ICICI Personal Financial Services Limited and ICICI Capital Services Limited, and ICICI Bank. The merger was approved by the ICICI and ICICI Bank shareholders in January 2002 by the Gujarat High Court in Ahmadabad in March 2002. The Supreme Court of the Mumbai Supreme Court and the Reserve Bank of India in April 
2002. Following the merger, ICICI Group support and banking operations, both for sale and sale, combined with one business.

The bank has state-owned companies in the United Kingdom, Russia and Canada; branches in the United States, Singapore, Bahrain, Hong Kong, Sri Lanka, Qatar, and the Daily International Finance Center; and representative offices in the United Arab Emirates, China, South Africa, Bangladesh, Thailand, Malaysia and Indonesia. The UK-owned company has set up branches in Belgium and Germany.

ICICI Bank provides a wide range of banking products and financial services to corporate and retail clients with a variety of delivery and its specialized services in the areas of investment banking, health and non-life insurance, business finance, and asset management.

The services offered include:

- Personal Banking

- Privilege Banking

- Wealth Management

- NRI Banking

- Corporate Banking

- Business Banking

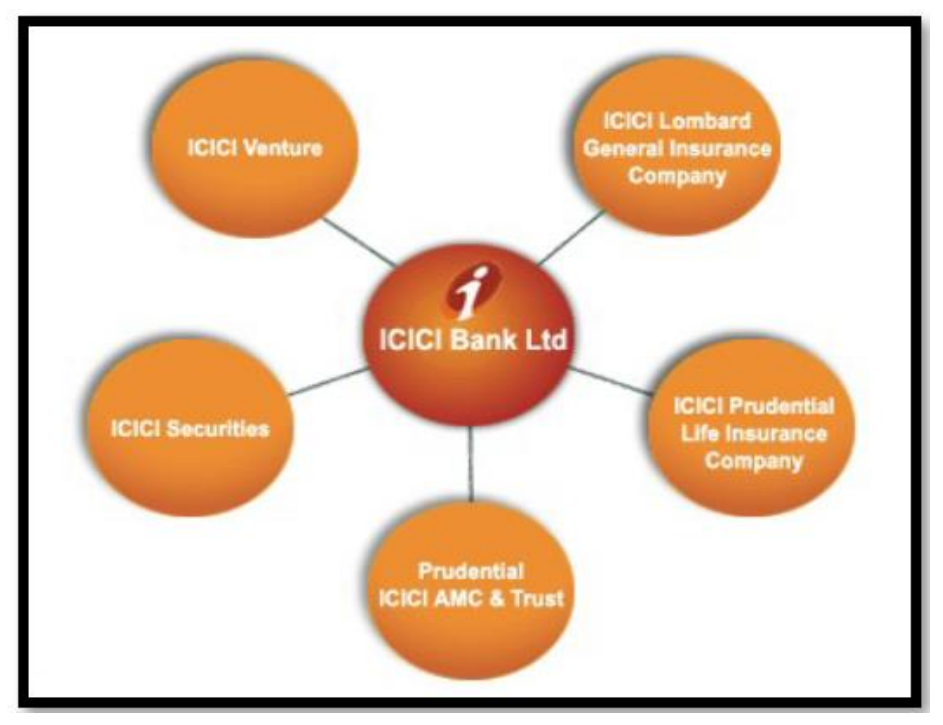

Figure 1

\section{Capital Structure}

The Authorized Capital of ICICI Bank is 214.75 Crores. The Issued, Subscribed, and Paid-up Capital is divided into 1113250642 equity shares @ Rs.10/- each.

\subsection{Vision and Mission of ICICI Bank}

\section{VISION}

To be the leading provider of financial services in India and a global central bank.

\section{MISSION}

To leverage our people, technology, speed, and financial capital to: 
- Be the banker of our customers' first choice by delivering high-quality, world-class products and services.

- Expand the frontiers of our business globally.

- Play a proactive role in the full realization of India's potential.

- Maintain a healthy financial profile and allocate our income to every business and area.

- Maintaining high standards of management and ethics.

- Contribute positively to the various countries and markets we operate.

- Build stakeholder participation.

\section{LITERATURE REVIEW}

- Gupta Shashi K. The establishment of ICICI aims to fill specific institutional institutions' gaps to finance industrial activities in the private sector. It will also serve as a fund for the development of the industry.

- Aggarwal Nisha, Gupta Neeti ICICI provides comprehensive assistance in building, expanding, and revitalizing industrial enterprises in India's private sector and encourages private financial participation, both internally and externally, in such businesses.

- Khan MY ICICI Ltd. (and its two services, ICICI Personal Finance Services Ltd. and ICICI Capital Services Ltd.) have been merged with ICICI bank Ltd, as of May 3, 2002. At that time, DFI is no longer operating there. Its primary purpose is to promote and encourage private investment in industrial investment and to increase investment markets.

- Bhole L. M. ICICI Bank is the largest private bank in India. Provides a variety of financial services at both corporate and sales levels. Since the mid-1990s, ICICI has been developing the necessary sub-companies and expanding services that will allow it to become a "global bank." In 1999-2000, business finance increased to $47 \%$ of ICICI's total lending portfolio from $36 \%$ in 1998-99.

\section{METHODOLOGY}

Required data for this project are collected from secondary sources.

- The data is collected from secondary sources.

- The financial data and information are gathered from the annual reports of the company.

- The data collected was analyzed through a comparative Balance sheet, profit and loss ratio.

- The results are presented using tables, graphs along with their interpretations.

I have taken the following secondary data:

- The balance sheet of ICICI Bank.

- Profit and loss account of ICICI Bank.

The tools used for the study are:

- Comparative financial statements

- Ratio analysis

- Common size statements 


\section{RESULTS AND DISCUSSIONS}

\subsection{Financial Performance Analysis \\ Comparative Balance Sheet}

Table 1

\begin{tabular}{|c|c|c|c|c|c|}
\hline \multicolumn{3}{|c|}{ Consolidated Balance Sheet } & \multicolumn{3}{|c|}{ - in Rs. Cr. } \\
\hline Years & March 2019 & March 2018 & March 2017 & March 2016 & March 2015 \\
\hline \multicolumn{6}{|c|}{ Capital and Liabilities: } \\
\hline Equity Share Capital & $1,289.46$ & $1,285.81$ & $1,165.11$ & $1,163.17$ & $1,159.66$ \\
\hline $\begin{array}{l}\text { Employee Stock } \\
\text { Option }\end{array}$ & 4.68 & 5.57 & 6.26 & 6.7 & 7.44 \\
\hline Reserves & $1,09,889.27$ & $1,06,310.68$ & $1,00,395.52$ & $90,123.37$ & $83,537.44$ \\
\hline Net Worth & $1,11,183.41$ & $1,07,602.06$ & $1,01,566.89$ & $91,293.24$ & $84,704.54$ \\
\hline Deposits & $6,81,316.94$ & $5,85,796.11$ & $5,12,587.26$ & $4,51,077.39$ & $3,85,955.25$ \\
\hline Borrowings & $2,10,324.12$ & $2,29,401.83$ & $1,88,286.76$ & $2,20,377.66$ & $2,11,252.00$ \\
\hline Total Debt & $8,91,641.06$ & $8,15,197.94$ & $7,00,874.02$ & $6,71,455.05$ & $5,97,207.25$ \\
\hline Minority Interest & $6,580.54$ & $6,008.19$ & $4,865.31$ & $3,355.64$ & $2,505.81$ \\
\hline Policy Holders Funds & $1,52,378.75$ & 0 & 0 & 0 & 0 \\
\hline $\begin{array}{l}\text { Other Liabilities \& } \\
\text { Provisions }\end{array}$ & $73,940.14$ & $1,92,445.22$ & $1,75,353.32$ & $1,49,834.79$ & $1,41,661.56$ \\
\hline Total Liabilities & $12,35,723.90$ & $11,21,253.41$ & $9,82,659.54$ & $9,15,938.72$ & $8,26,079.16$ \\
\hline \multicolumn{6}{|c|}{ Assets } \\
\hline $\begin{array}{l}\text { Cash \& Balances with } \\
\text { RBI }\end{array}$ & $38,066.28$ & $33,272.60$ & $31,891.26$ & $27,277.56$ & $25,837.67$ \\
\hline $\begin{array}{l}\text { Balance with Banks, } \\
\text { Money at Call }\end{array}$ & $49,324.62$ & $55,726.53$ & $48,599.61$ & $37,758.41$ & $21,799.50$ \\
\hline Advances & $6,46,961.68$ & $5,66,854.22$ & $5,15,317.31$ & $4,93,729.11$ & $4,38,490.10$ \\
\hline Investments & $3,98,200.76$ & $3,72,207.68$ & $3,04,373.29$ & $2,86,044.09$ & $3,02,761.63$ \\
\hline Gross Block & $9,660.42$ & $9,465.01$ & $9,337.96$ & $8,713.46$ & $5,871.21$ \\
\hline Revaluation Reserves & $3,070.00$ & $3,027.64$ & $3,065.11$ & $2,817.47$ & 0 \\
\hline Net Block & $6,590.42$ & $6,437.37$ & $6,272.85$ & $5,895.99$ & $5,871.21$ \\
\hline Other Assets & $96,580.14$ & $86,755.00$ & $76,205.22$ & $65,233.57$ & $31,319.07$ \\
\hline Total Assets & $12,35,723.90$ & $11,21,253.40$ & $9,82,659.54$ & $9,15,938.73$ & $8,26,079.18$ \\
\hline Contingent Liabilities & $26,61,651.12$ & $14,52,896.94$ & $8,68,410.51$ & $11,82,880.58$ & $10,35,330.05$ \\
\hline Book Value (Rs) & 172.47 & 167.39 & 174.37 & 157.47 & 146.68 \\
\hline
\end{tabular}

\subsection{Profit and Loss Account of ICICI Bank Ltd}

Table 2

Standalone Profit \& Loss account

\begin{tabular}{|l|c|c|c|c|c|}
\hline \multicolumn{1}{|c|}{ Years } & Mar-19 & Mar-18 & Mar-17 & Mar-16 & Mar-15 \\
\hline Income & & & & & \\
\hline Interest Earned & $63,401.19$ & $54,965.89$ & $54,156.28$ & $52,739.43$ & $49,091.14$ \\
\hline Other Income & $14,512.16$ & $17,419.63$ & $19,504.48$ & $15,323.05$ & $12,176.13$ \\
\hline Total Income Expenditure & $77,913.35$ & $72,385.52$ & $73,660.76$ & $68,062.48$ & $61,267.27$ \\
\hline \multicolumn{2}{|l|}{} & & & & \\
\hline Interest expended & $36,386.40$ & $31,940.05$ & $32,418.96$ & $31,515.39$ & $30,051.53$ \\
\hline Employee Cost & $6,808.24$ & $5,913.95$ & $5,733.71$ & $3,012.69$ & $4,749.88$ \\
\hline $\begin{array}{l}\text { Selling, Admin \& Misc. } \\
\text { Expenses }\end{array}$ & $50,239.65$ & $28,158.48$ & $24,862.72$ & $34,777.42$ & $15,092.36$ \\
\hline Depreciation & 776.91 & 780.74 & 757.65 & 698.51 & 658.95 \\
\hline Operating Expenses & $37,750.20$ & $16,889.06$ & $14,668.42$ & $24,351.37$ & $11,956.63$ \\
\hline
\end{tabular}


Study on Financial Analysis of ICICI Bank

\begin{tabular}{|l|c|c|c|c|c|}
\hline Provisions \& Contingencies & $20,074.60$ & $17,964.11$ & $16,685.66$ & $14,137.25$ & $8,544.56$ \\
\hline Total Expenses & $94,211.20$ & $66,793.22$ & $63,773.04$ & $70,004.01$ & $50,552.72$ \\
\hline Net Profit for the Year & $-16,297.84$ & $5,592.30$ & $9,887.72$ & $-1,941.53$ & $10,714.56$ \\
\hline Profit brought forward & $18,495.26$ & $18,744.94$ & $17,132.19$ & $17,261.42$ & $13,318.59$ \\
\hline Total & $2,197.42$ & $24,337.24$ & $27,019.91$ & $15,319.89$ & $24,033.15$ \\
\hline Equity Dividend & 965.13 & $1,457.46$ & 0.95 & $2,907.52$ & $2,898.81$ \\
\hline Corporate Dividend Tax & 0 & 8.73 & -7.19 & 279.37 & 271.15 \\
\hline \multicolumn{2}{|c|}{ Per-share data (annualized) } & & & & \\
\hline Earnings Per Share (Rs) & -25.28 & 8.7 & 16.98 & -3.34 & 18.48 \\
\hline Equity Dividend (\%) & 50 & 75 & 125 & 250 & 250 \\
\hline Book Value (Rs) Appropriations & 163.38 & 158.91 & 166.37 & 149.47 & 138.72 \\
\hline \multicolumn{2}{|c|}{} & & & & \\
\hline $\begin{array}{l}\text { Transfer to Statutory } \\
\text { Reserves }\end{array}$ & $3,013.86$ & $5,560.91$ & $8,194.58$ & $6,668.62$ & $4,062.57$ \\
\hline Transfer to Other Reserves & 0 & 0.01 & 0 & 0.01 & 0 \\
\hline $\begin{array}{l}\text { Proposed Dividend/Transfer } \\
\text { to Govt. }\end{array}$ & 965.13 & $1,466.19$ & -6.24 & $3,186.89$ & $3,169.96$ \\
\hline Balance c/f to Balance Sheet & $17,879.57$ & $18,495.26$ & $18,744.94$ & $17,132.19$ & $17,261.42$ \\
\hline \begin{tabular}{l} 
Total \\
\hline
\end{tabular} & $21,858.56$ & $25,522.37$ & $26,933.28$ & $26,987.71$ & $24,493.95$ \\
\hline
\end{tabular}

\section{FINANCIAL STATEMENT ANALYSIS}

\subsection{Comparative Balance Sheet of ICICI Bank from 2015-2016 to 2018-2019}

Table 3

\begin{tabular}{|c|c|c|c|c|c|c|c|c|}
\hline \multirow{3}{*}{ Particulars } & & & & & & & \multirow{2}{*}{\multicolumn{2}{|c|}{$\frac{\text { (Rs. In Crores) }}{2018-2019}$}} \\
\hline & \multicolumn{2}{|c|}{ 2015-2016 } & \multicolumn{2}{|c|}{ 2016-2017 } & \multicolumn{2}{|c|}{ 2017-2018 } & & \\
\hline & $\begin{array}{c}\text { Absolute } \\
\text { Change }\end{array}$ & $\begin{array}{c}\% \text { of } \\
\text { Absolute } \\
\text { Change }\end{array}$ & $\begin{array}{l}\text { Absolute } \\
\text { Change }\end{array}$ & $\begin{array}{c}\% \text { of } \\
\text { Absolute } \\
\text { Change }\end{array}$ & $\begin{array}{l}\text { Absolute } \\
\text { Change }\end{array}$ & $\begin{array}{c}\% \text { of } \\
\text { Absolute } \\
\text { Change }\end{array}$ & $\begin{array}{c}\text { Absolute } \\
\text { Change }\end{array}$ & $\begin{array}{c}\% \text { of } \\
\text { Absolute } \\
\text { Change }\end{array}$ \\
\hline $\begin{array}{l}\text { Capital and } \\
\text { Liabilities }\end{array}$ & & & & & & & & \\
\hline $\begin{array}{l}\text { Total Share } \\
\text { Capital }\end{array}$ & 3.51 & 0.3027 & 1.94 & 0.1668 & 120.70 & 10.36 & 3.65 & 0.2839 \\
\hline $\begin{array}{l}\text { Equity Share } \\
\text { Capital }\end{array}$ & 3.51 & 0.3027 & 1.94 & 0.1668 & 120.70 & 10.36 & 3.65 & 0.2839 \\
\hline $\begin{array}{l}\text { Employee } \\
\text { Stock Option }\end{array}$ & -0.74 & -9.946 & -0.44 & -6.5672 & -0.69 & -11.02 & -0.89 & -15.98 \\
\hline Reserves & $6,585.93$ & 7.8838 & $10,272.15$ & 11.398 & $5,915.16$ & 5.892 & $3,578.59$ & 3.3662 \\
\hline Net Worth & $6,588.70$ & 7.7784 & $10,273.65$ & 11.253 & $6,035.17$ & 5.942 & $3,581.35$ & 3.3283 \\
\hline Deposits & $65,122.14$ & 16.873 & $61,509.87$ & 13.636 & $73,208.85$ & 14.28 & $95,520.83$ & 16.306 \\
\hline Borrowings & $9,125.66$ & 4.3198 & $-32,090.90$ & -14.562 & $41,115.07$ & 21.84 & $-19,077.71$ & -8.316 \\
\hline Total Debt & $74,247.80$ & 12.433 & $29,418.97$ & 4.3814 & $1,14,323.92$ & 16.31 & $76,443.12$ & 9.3772 \\
\hline $\begin{array}{l}\text { Minority } \\
\text { Interest }\end{array}$ & 849.83 & 33.914 & $1,509.67$ & 44.989 & $1,142.88$ & 23.49 & 572.35 & 9.5262 \\
\hline $\begin{array}{l}\text { Policy } \\
\text { Holders } \\
\text { Funds } \\
\end{array}$ & 0.00 & 0.00 & 0.00 & 0.00 & 0.00 & 0.00 & $1,52,378.75$ & 0.00 \\
\hline $\begin{array}{l}\text { Other } \\
\text { Liabilities \& } \\
\text { Provisions }\end{array}$ & $8,173.23$ & 5.7695 & $25,518.53$ & 17.031 & $17,091.90$ & 9.747 & $-1,18,505.08$ & -61.58 \\
\hline $\begin{array}{l}\text { Total Capital } \\
\text { and } \\
\text { Liabilities }\end{array}$ & $89,859.56$ & 10.878 & $66,720.82$ & 7.2844 & $1,38,593.87$ & 14.1 & $1,14,470.49$ & 10.209 \\
\hline ASSETS & & & & & & & & \\
\hline $\begin{array}{l}\text { Cash \& } \\
\text { Balances } \\
\text { with RBI } \\
\end{array}$ & $1,439.89$ & 5.5728 & $4,613.70$ & 16.914 & $1,381.34$ & 4.331 & $4,793.68$ & 14.407 \\
\hline $\begin{array}{l}\text { Balance with } \\
\text { Banks, } \\
\text { Money at } \\
\text { Call }\end{array}$ & $15,958.91$ & 73.208 & $10,841.20$ & 28.712 & $7,126.92$ & 14.66 & $-6,401.91$ & -11.49 \\
\hline Advances & $55,239.01$ & 12.598 & $21,588.20$ & 4.3725 & $51,536.91$ & 10 & $80,107.46$ & 14.132 \\
\hline Investments & $-16,717.54$ & -5.522 & $18,329.20$ & 6.4078 & $67,834.39$ & 22.29 & $25,993.08$ & 6.9835 \\
\hline
\end{tabular}


K. Neelavathi, Ramya Chavali and Rohan Bhavirisetty

\begin{tabular}{|l|c|c|c|c|c|c|c|c|}
\hline Gross Block & $2,842.25$ & 48.41 & 624.50 & 7.1671 & 127.05 & 1.361 & 195.41 & 2.0646 \\
\hline $\begin{array}{l}\text { Revaluation } \\
\text { Reserves }\end{array}$ & $2,817.47$ & 0 & 247.64 & 8.7894 & -37.47 & -1.222 & 42.36 & 1.3991 \\
\hline Net Block & 24.78 & 0.4221 & 376.86 & 6.3918 & 164.52 & 2.623 & 153.05 & 2.3775 \\
\hline Other Assets & $33,914.50$ & 108.29 & $10,971.65$ & 16.819 & $10,549.78$ & 13.84 & $9,825.14$ & 11.325 \\
\hline Total Assets & $89,859.55$ & 10.878 & $66,720.81$ & 7.2844 & $1,38,593.86$ & 14.1 & $1,14,470.50$ & 10.209 \\
\hline
\end{tabular}

\section{Interpretation}

1.The company's total capital for 2015-16 was $10.878 \%$, and for the year 2016-17, it reduced to $7.2844 \%$. But in the year 2017-18 it increased to $14.1 \%$ and in the current year $2018-19$ it again declined to $10.209 \%$, this shows that the company has a good amount of capital and the liabilities of the company are also stabilized.

2. The company's total assets for the year 2015-16 were $10.878 \%$, i.e., 89859.56 , and for the year 2016-17, it reduced to $7.2844 \%$, i.e., 66720.82. But in the year 2017-18, it increased to $14.1 \%$, i.e., 138593.87 , and in the current year 2018-19, it again reduced to $10.209 \%$, i.e., 114470.49 , this shows that the company has maintained its assets as well as its liabilities.

Table 4

\begin{tabular}{|c|c|c|c|c|c|c|c|c|}
\hline \multirow{3}{*}{ Particulars } & & & & & & & \multirow{2}{*}{\multicolumn{2}{|c|}{$\begin{array}{l}\text { (Rs. In Crores) } \\
\mathbf{2 0 1 8 - 2 0 1 9} \\
\end{array}$}} \\
\hline & \multicolumn{2}{|l|}{ 2015-2016 } & \multicolumn{2}{|l|}{ 2016-2017 } & \multicolumn{2}{|l|}{$2017-2018$} & & \\
\hline & $\begin{array}{l}\text { Absolute } \\
\text { Change }\end{array}$ & $\begin{array}{l}\% \text { of } \\
\text { Absolute } \\
\text { Change }\end{array}$ & $\begin{array}{l}\text { Absolute } \\
\text { Change }\end{array}$ & $\begin{array}{l}\text { \% of } \\
\text { Absolute } \\
\text { Change }\end{array}$ & $\begin{array}{l}\text { Absolute } \\
\text { Change }\end{array}$ & $\begin{array}{l}\text { \% of } \\
\text { Absolute } \\
\text { Change }\end{array}$ & $\begin{array}{l}\text { Absolute } \\
\text { Change }\end{array}$ & $\begin{array}{l}\% \text { of } \\
\text { Absolute } \\
\text { Change }\end{array}$ \\
\hline \multicolumn{9}{|l|}{ Income } \\
\hline Interest Earned & $3,648.29$ & 7.4317 & $1,416.85$ & 2.6865 & 809.61 & 1.495 & $8,435.30$ & 15.346 \\
\hline Other Income & $3,146.92$ & 25.845 & $4,181.43$ & 27.288 & $-2,084.85$ & -10.689 & $-2,907.47$ & -16.69 \\
\hline Total Income & $6,795.21$ & 11.091 & $5,598.28$ & 8.2252 & $-1,275.24$ & -1.7312 & $5,527.83$ & 7.6367 \\
\hline \multicolumn{9}{|l|}{ Expenditure } \\
\hline $\begin{array}{l}\text { Interest } \\
\text { expended }\end{array}$ & $1,463.86$ & 4.8712 & 903.57 & 2.8671 & -478.91 & -1.4773 & $4,446.35$ & 13.921 \\
\hline Employee Cost & $-1,737.19$ & -36.57 & $2,721.02$ & 90.319 & 180.24 & 3.1435 & 894.29 & 15.122 \\
\hline $\begin{array}{l}\text { Selling, Admin } \\
\text { \& Misc. } \\
\text { Expenses }\end{array}$ & $19,685.06$ & 130.43 & $-9,914.70$ & -28.51 & $3,295.76$ & 13.256 & $22,081.17$ & 78.417 \\
\hline Depreciation & 39.56 & 6.0035 & 59.14 & 8.4666 & 23.09 & 3.0476 & -3.83 & -0.491 \\
\hline $\begin{array}{l}\text { Operating } \\
\text { Expenses }\end{array}$ & $12,394.74$ & 103.66 & $-9,682.95$ & -39.76 & $2,220.64$ & 15.139 & $20,861.14$ & 123.52 \\
\hline $\begin{array}{l}\text { Provisions \& } \\
\text { Contingencies }\end{array}$ & $5,592.69$ & 65.453 & $2,548.41$ & 18.026 & $1,278.45$ & 7.662 & $2,110.49$ & 11.748 \\
\hline Total Expenses & $19,451.29$ & 38.477 & $-6,230.97$ & -8.901 & $3,020.18$ & 4.7358 & $27,417.98$ & 41.049 \\
\hline $\begin{array}{l}\text { Net Profit for } \\
\text { the Year }\end{array}$ & $-12,656.09$ & -118.1 & $11,829.25$ & -609.3 & $-4,295.42$ & -43.442 & $-21,890.14$ & -391.4 \\
\hline $\begin{array}{l}\text { Profit brought } \\
\text { forward }\end{array}$ & $3,942.83$ & 29.604 & -129.23 & -0.749 & $1,612.75$ & 9.4136 & -249.68 & -1.332 \\
\hline Total & $-8,713.26$ & -36.26 & $11,700.02$ & 76.371 & $-2,682.67$ & -9.9285 & $-22,139.82$ & -90.97 \\
\hline
\end{tabular}

The Above table is the Comparative Profit and loss account of ICICI Bank From 2015-2016 To 2018-2019, and its interpretation is made below.

\section{Interpretation}

1. The company's total income for $2015-16$ was $11.091 \%$, i.e., 6795.21 , and 2016-17 was $8.2252 \%$, i.e., 5598.28 . In the year $2017-18$, it reduced to $1.7312 \%$, i.e., 1275.24 , and the following year, $2018-19$, it comes up to $7.6367 \%$, i.e., 5527.83 means that the income was increasing for the company in the year 2018-19.

2. The company's total expenses for the year 2015-16 were $38.477 \%$, i.e., 19451.29 , but in 2016-17, the expenses of the company were reduced to $-8.901 \%$, i.e., -6230.97 . For the year $2017-18$, the company's expenses again came up to $4.7358 \%$, i.e., 3020.18 , and in the year 
2018-19, the expenses came up also to $41.049 \%$, i.e., 27417.98 , which means the expenses of the company were increasing. This shows that the company is not working correctly.

\subsection{Ratio Analysis}

\section{Current Ratios}

\begin{tabular}{|c|c|}
\hline Years & Current Ratios \\
\hline 2015 & 0.06 \\
\hline 2016 & 0.13 \\
\hline 2017 & 0.12 \\
\hline 2018 & 0.12 \\
\hline 2019 & 0.12 \\
\hline
\end{tabular}

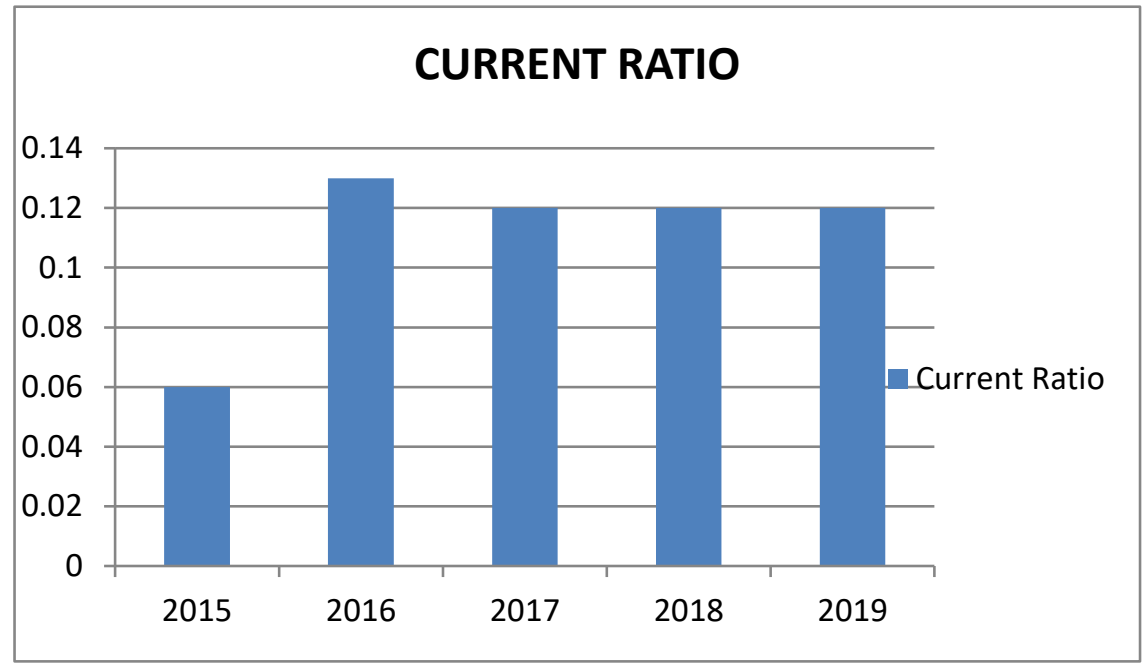

Figure 1

\section{Quick Ratios}

\begin{tabular}{|c|c|}
\hline Years & Quick Ratios \\
\hline 2015 & 13.81 \\
\hline 2016 & 14.97 \\
\hline 2017 & 16.31 \\
\hline 2018 & 20.44 \\
\hline 2019 & 18.66 \\
\hline
\end{tabular}

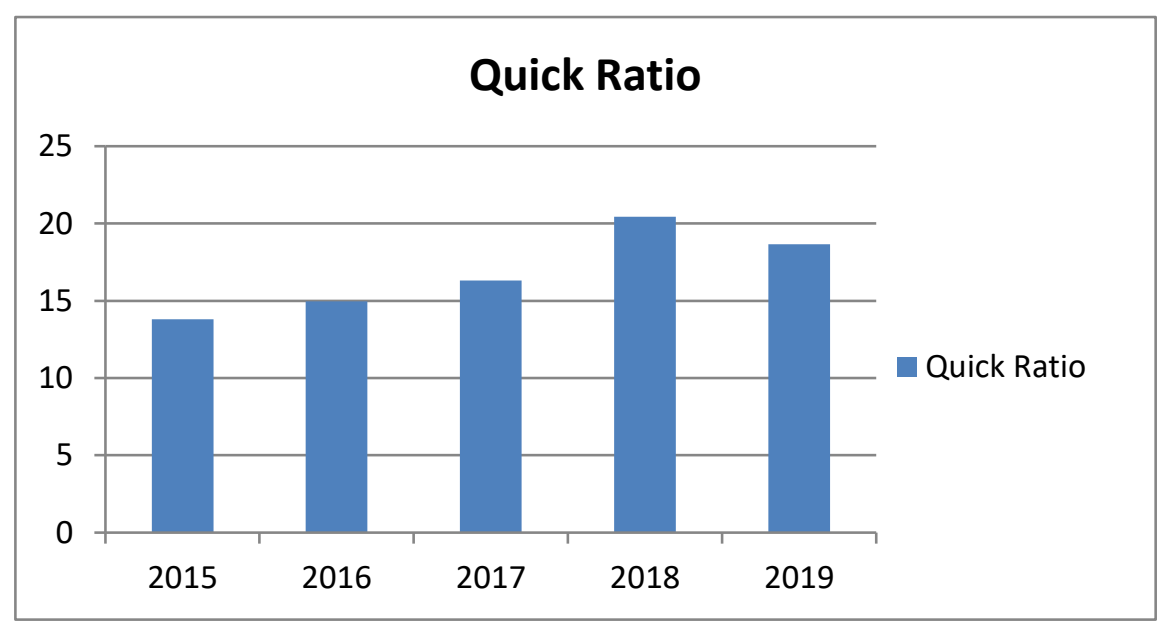

Figure 2 


\section{Debt to Equity Ratio}

\begin{tabular}{|c|c|}
\hline Years & Debt to Equity Ratio \\
\hline 2015 & 6.95 \\
\hline 2016 & 0.87 \\
\hline 2017 & 0.82 \\
\hline 2018 & 0.90 \\
\hline 2019 & 0.86 \\
\hline
\end{tabular}

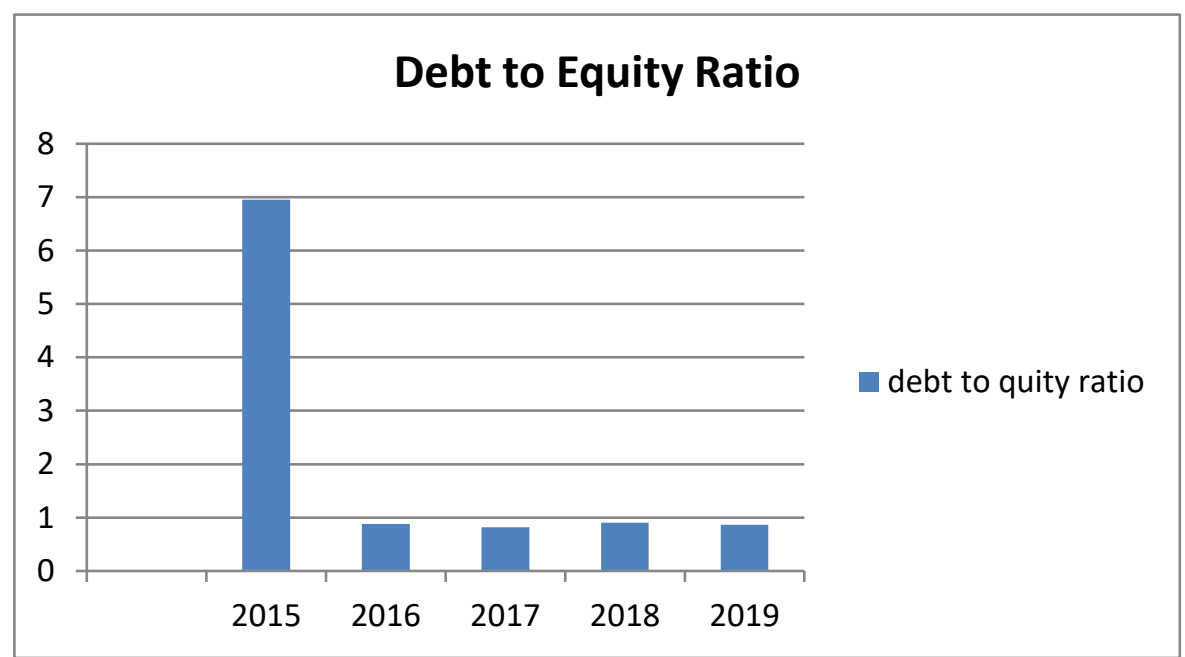

Figure 3

\section{Total Assets to Debt Ratio}

\begin{tabular}{|c|c|}
\hline Years & Total Assets to Debt Ratio \\
\hline 2015 & 1.38 \\
\hline 2016 & 1.36 \\
\hline 2017 & 1.40 \\
\hline 2018 & 1.37 \\
\hline 2019 & 1.38 \\
\hline
\end{tabular}

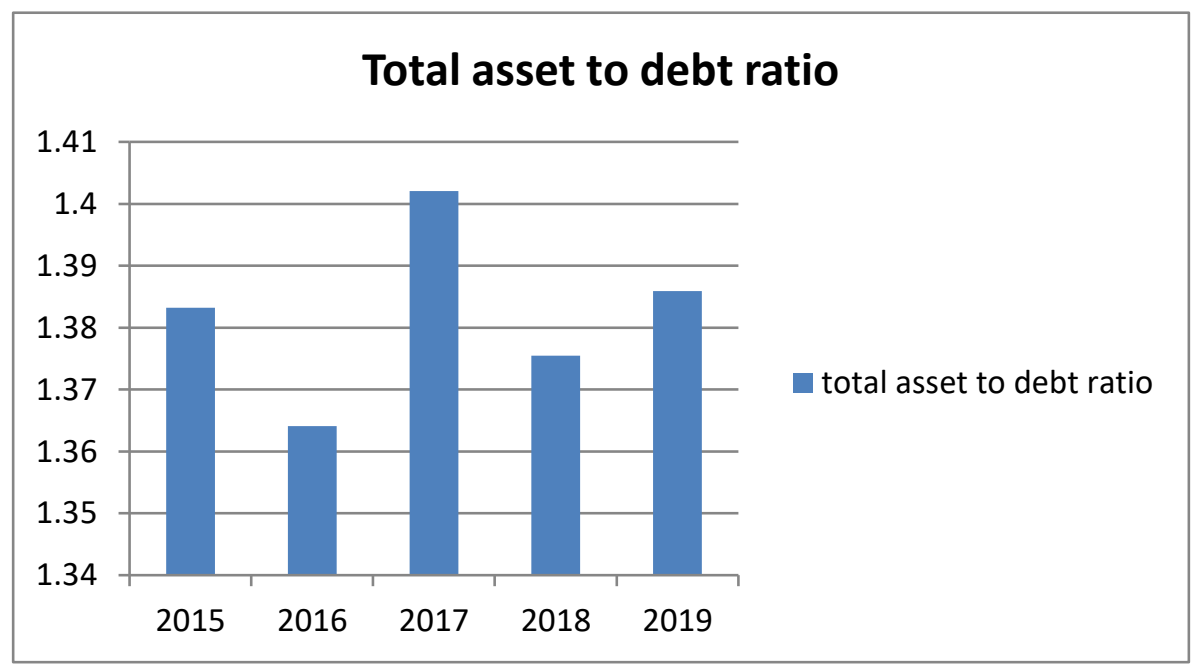

Figure 4 


\section{Proprietary Ratios}

\begin{tabular}{|c|c|}
\hline Years & Proprietary Ratio \\
\hline 2015 & 9.75 \\
\hline 2016 & 10.03 \\
\hline 2017 & 9.67 \\
\hline 2018 & 10.42 \\
\hline 2019 & 11.11 \\
\hline
\end{tabular}

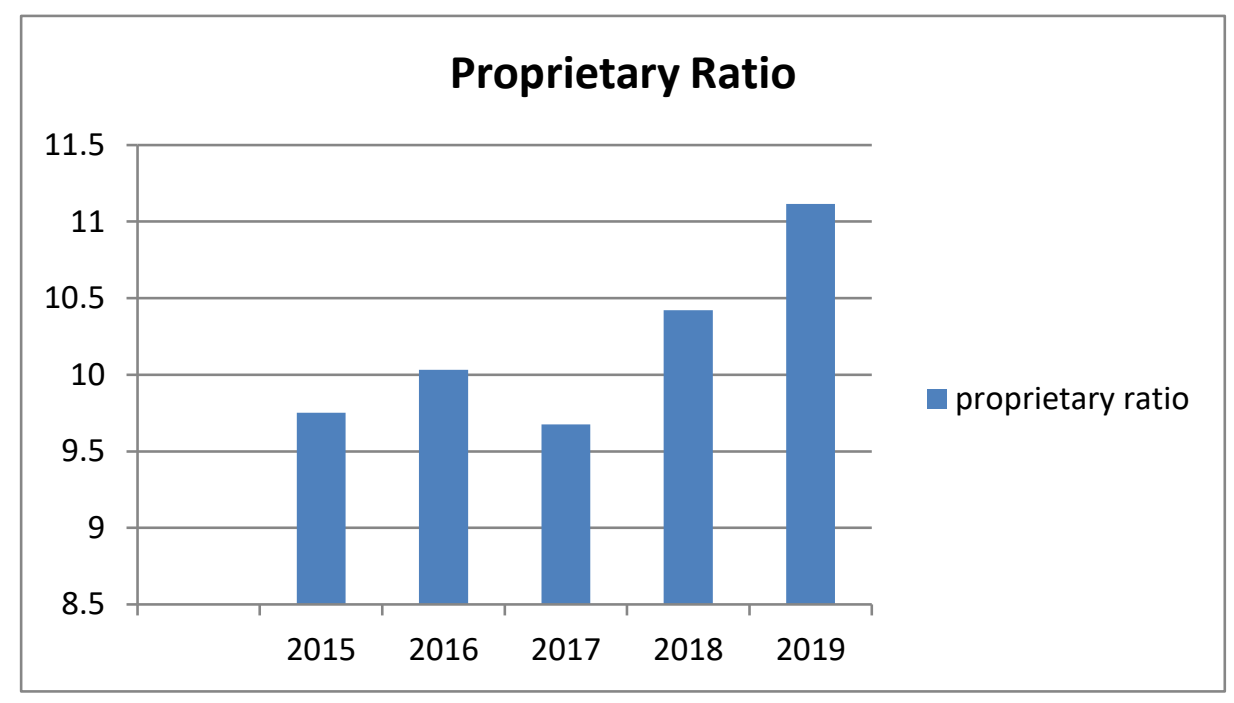

Figure 5

\section{Interpretation}

1.The company's quick ratio for the year 2018-19 is 18.66 are the current ratio of the company is 0.12 . This shows that the company is liable to pay its debts, and there is immediate liquidity in the company.

2. The company's debt-to-equity ratio for the year 2018-19 is 0.866419013 , which shows that the company has a good grip on its equity and the debts are also under control.

3. The company's total asset to debt ratio for the year 2018-19 is 1.385898379 , which shows that the company has an enormous investment value and the debt is under its control.

4. The company's proprietary ratio for $2018-19$ is 11.11428315 , which shows that its financial strength is good.

\subsection{Common Size Balance Sheet of ICICI Bank from 2015-2016 to 2018-2019}

\begin{tabular}{|c|c|c|c|c|c|c|c|c|}
\hline \multirow{3}{*}{ Particulars } & & & & & & & (Rs. In & cores) \\
\hline & \multicolumn{2}{|c|}{ 2015-2016 } & \multicolumn{2}{|c|}{ 2016-2017 } & \multicolumn{2}{|c|}{ 2017-2018 } & \multicolumn{2}{|c|}{ 2018-2019 } \\
\hline & $\begin{array}{c}\text { Absolute } \\
\text { Change }\end{array}$ & $\begin{array}{c}\% \text { of } \\
\text { Absolute } \\
\text { Change } \\
\end{array}$ & $\begin{array}{l}\text { Absolute } \\
\text { Change }\end{array}$ & $\begin{array}{c}\% \text { of } \\
\text { Absolute } \\
\text { Change } \\
\end{array}$ & $\begin{array}{c}\text { Absolute } \\
\text { Change }\end{array}$ & $\begin{array}{c}\% \text { of } \\
\text { Absolute } \\
\text { Change } \\
\end{array}$ & $\begin{array}{l}\text { Absolute } \\
\text { Change }\end{array}$ & $\begin{array}{c}\% \text { of } \\
\text { Absolute } \\
\text { Change }\end{array}$ \\
\hline $\begin{array}{l}\text { Capital and } \\
\text { Liabilities }\end{array}$ & & & & & & & & \\
\hline $\begin{array}{l}\text { Total Share } \\
\text { Capital }\end{array}$ & 3.51 & 0.3027 & 1.94 & 0.1668 & 120.70 & 10.36 & 3.65 & 0.2839 \\
\hline $\begin{array}{l}\text { Equity Share } \\
\text { Capital }\end{array}$ & 3.51 & 0.3027 & 1.94 & 0.1668 & 120.70 & 10.36 & 3.65 & 0.2839 \\
\hline
\end{tabular}


K. Neelavathi, Ramya Chavali and Rohan Bhavirisetty

\begin{tabular}{|c|c|c|c|c|c|c|c|c|}
\hline $\begin{array}{l}\text { Employee } \\
\text { Stock Option }\end{array}$ & -0.74 & -9.946 & -0.44 & -6.5672 & -0.69 & -11.02 & -0.89 & -15.98 \\
\hline Reserves & $6,585.93$ & 7.8838 & $10,272.15$ & 11.398 & $5,915.16$ & 5.892 & $3,578.59$ & 3.3662 \\
\hline Net Worth & $6,588.70$ & 7.7784 & $10,273.65$ & 11.253 & $6,035.17$ & 5.942 & $3,581.35$ & 3.3283 \\
\hline Deposits & $65,122.14$ & 16.873 & $61,509.87$ & 13.636 & $73,208.85$ & 14.28 & $95,520.83$ & 16.306 \\
\hline Borrowings & $9,125.66$ & 4.3198 & $\begin{array}{c}- \\
32,090.90 \\
\end{array}$ & -14.562 & $41,115.07$ & 21.84 & $-19,077.71$ & -8.316 \\
\hline Total Debt & $74,247.80$ & 12.433 & $29,418.97$ & 4.3814 & $1,14,323.92$ & 16.31 & $76,443.12$ & 9.3772 \\
\hline $\begin{array}{l}\text { Minority } \\
\text { Interest }\end{array}$ & 849.83 & 33.914 & $1,509.67$ & 44.989 & $1,142.88$ & 23.49 & 572.35 & 9.5262 \\
\hline $\begin{array}{l}\text { Policy Holders } \\
\text { Funds }\end{array}$ & 0.00 & 0.00 & 0.00 & 0.00 & 0.00 & 0.00 & $1,52,378.75$ & 0.00 \\
\hline $\begin{array}{l}\text { Other } \\
\text { Liabilities \& } \\
\text { Provisions }\end{array}$ & $8,173.23$ & 5.7695 & $25,518.53$ & 17.031 & $17,091.90$ & 9.747 & $\stackrel{-}{-}$ & -61.58 \\
\hline $\begin{array}{l}\text { Total Capital } \\
\text { and Liabilities }\end{array}$ & $89,859.56$ & 10.878 & $66,720.82$ & 7.2844 & $1,38,593.87$ & 14.1 & $1,14,470.49$ & 10.209 \\
\hline ASSETS & & & & & & & & \\
\hline $\begin{array}{l}\text { Cash \& } \\
\text { Balances with } \\
\text { RBI }\end{array}$ & $1,439.89$ & 5.5728 & $4,613.70$ & 16.914 & $1,381.34$ & 4.331 & $4,793.68$ & 14.407 \\
\hline $\begin{array}{l}\text { Balance with } \\
\text { Banks, Money } \\
\text { at Call }\end{array}$ & $15,958.91$ & 73.208 & $10,841.20$ & 28.712 & $7,126.92$ & 14.66 & $-6,401.91$ & -11.49 \\
\hline Advances & $55,239.01$ & 12.598 & $21,588.20$ & 4.3725 & $51,536.91$ & 10 & $80,107.46$ & 14.132 \\
\hline Investments & $\begin{array}{c}- \\
16,717.54\end{array}$ & -5.522 & $18,329.20$ & 6.4078 & $67,834.39$ & 22.29 & $25,993.08$ & 6.9835 \\
\hline Gross Block & $2,842.25$ & 48.41 & 624.50 & 7.1671 & 127.05 & 1.361 & 195.41 & 2.0646 \\
\hline $\begin{array}{l}\text { Revaluation } \\
\text { Reserves }\end{array}$ & $2,817.47$ & 0 & 247.64 & 8.7894 & -37.47 & -1.222 & 42.36 & 1.3991 \\
\hline Net Block & 24.78 & 0.4221 & 376.86 & 6.3918 & 164.52 & 2.623 & 153.05 & 2.3775 \\
\hline Other Assets & $33,914.50$ & 108.29 & $10,971.65$ & 16.819 & $10,549.78$ & 13.84 & $9,825.14$ & 11.325 \\
\hline Total Assets & $89,859.55$ & 10.878 & $66,720.81$ & 7.2844 & $1,38,593.86$ & 14.1 & $1,14,470.50$ & 10.209 \\
\hline
\end{tabular}

\section{Interpretation}

1. The company's total capital for $2015-16$ was $10.87 \%$, i.e., 89859.56 , and for the year 201617, it reduced to $7.28 \%$, i.e., 66720.82 . But in the year $2017-18$, it increased to $14.1 \%$, i.e., 138593.87.

2. In the current year 2018-19, it again reduced to $10.209 \%$, i.e., 114470.49 . This shows that the company has a good amount of capital, and the company's liabilities are being stabilized.

3. The Total Assets of the company for 2015-16 were 10.878\%, i.e., 89859.56, and for the year 2016-17, it reduced to 7.2844\%, i.e., 66720.82. But in the year 2017-18, it increased to $14.1 \%$, i.e., 138593.87, and in the current year 2018-19, it again reduced to $10.209 \%$, i.e., 114470.49 , this shows that the company has maintained its assets as well as its liabilities. 


\subsection{Common Size Statement of Profit \& Loss A/c of ICICI Bank from 2015-2016 to 2018-2019}

Table 6

\begin{tabular}{|c|c|c|c|c|c|c|c|c|c|c|}
\hline \multirow[t]{2}{*}{ Particulars } & \multicolumn{5}{|c|}{$\begin{array}{l}\text { ABSOLUTE AMOUNTS (Rs.) } \\
\end{array}$} & \multicolumn{5}{|c|}{$\begin{array}{c}\begin{array}{c}\text { Percentage of Revenue from Operation } \\
(\%)\end{array} \\
\end{array}$} \\
\hline & Mar-19 & Mar-18 & Mar-17 & Mar-16 & Mar-15 & $\begin{array}{l}\text { Mar- } \\
19\end{array}$ & $\begin{array}{c}\text { Mar- } \\
18\end{array}$ & $\begin{array}{c}\text { Mar- } \\
17\end{array}$ & $\begin{array}{c}\text { Mar- } \\
16\end{array}$ & $\underset{15}{\text { Mar- }}$ \\
\hline \multicolumn{11}{|l|}{ Income } \\
\hline $\begin{array}{l}\text { Interest } \\
\text { Earned }\end{array}$ & $63,401.19$ & $54,965.89$ & $54,156.28$ & $52,739.43$ & $49,091.14$ & 100.00 & 100.00 & 100.00 & 100.00 & 100.00 \\
\hline Other Income & $14,512.16$ & $17,419.63$ & $19,504.48$ & $15,323.05$ & $12,176.13$ & 22.89 & 31.69 & 36.02 & 29.05 & 24.80 \\
\hline Total Income & $77,913.35$ & 72385.52 & 73660.76 & 68062.48 & 61267.27 & 122.89 & 131.69 & 136.02 & 129.05 & 124.80 \\
\hline \multicolumn{11}{|l|}{ Expenditure } \\
\hline $\begin{array}{l}\text { Interest } \\
\text { expended }\end{array}$ & $36,386.40$ & $31,940.05$ & $32,418.96$ & $31,515.39$ & $30,051.53$ & 57.39 & 58.11 & 59.86 & 59.76 & 61.22 \\
\hline $\begin{array}{l}\text { Employee } \\
\text { Cost }\end{array}$ & $6,808.24$ & $5,913.95$ & $5,733.71$ & $3,012.69$ & $4,749.88$ & 10.74 & 10.76 & 10.59 & 5.71 & 9.68 \\
\hline $\begin{array}{l}\text { Selling, } \\
\text { Admin \& } \\
\text { Misc. } \\
\text { Expenses }\end{array}$ & $50,239.65$ & 28,158 & $24,862.72$ & & & & & & & \\
\hline Depreciation & 776.91 & 780.74 & 757.65 & 698.51 & 658.95 & 1.23 & 1.42 & 1.40 & 1.32 & 1.34 \\
\hline $\begin{array}{l}\text { Operating } \\
\text { Expenses }\end{array}$ & $37,750.20$ & $16,889.06$ & $14,668.42$ & $24,351.37$ & $11,956.63$ & 59.54 & 30.73 & 27.09 & 46.17 & 24.36 \\
\hline $\begin{array}{l}\text { Provisions \& } \\
\text { Contingencies }\end{array}$ & $20,074.60$ & $17,964.11$ & $16,685.66$ & $14,137.25$ & $8,544.56$ & 31.66 & 32.68 & 30.81 & 26.81 & 17.41 \\
\hline $\begin{array}{l}\text { Total } \\
\text { Expenses }\end{array}$ & $94,211.20$ & $66,793.22$ & $63,773.04$ & $70,004.01$ & $50,552.72$ & 148.60 & 121.52 & 117.76 & 132.74 & 102.98 \\
\hline $\begin{array}{l}\text { Net Profit for } \\
\text { the Year }\end{array}$ & $\begin{array}{c}- \\
16,297.84 \\
\end{array}$ & $5,592.30$ & $9,887.72$ & $-1,941.53$ & $10,714.56$ & -25.71 & 10.17 & 18.26 & -3.68 & 21.83 \\
\hline $\begin{array}{l}\text { Profit brought } \\
\text { forward }\end{array}$ & $18,495.26$ & $18,744.94$ & $17,132.19$ & $17,261.42$ & $13,318.59$ & 29.17 & 34.10 & 31.63 & 32.73 & 27.13 \\
\hline Total & $2,197.42$ & $24,337.24$ & $27,019.91$ & $15,319.89$ & $24,033.15$ & 3.47 & 44.28 & 49.89 & 29.05 & 48.96 \\
\hline $\begin{array}{l}\text { Equity } \\
\text { Dividend }\end{array}$ & 965.13 & $1,457.46$ & 0.95 & $2,907.52$ & $2,898.81$ & 1.52 & 2.65 & 0.00 & 5.51 & 5.90 \\
\hline $\begin{array}{l}\text { Corporate } \\
\text { Dividend Tax }\end{array}$ & 0 & 8.73 & -7.19 & 279.37 & 271.15 & 0.00 & 0.02 & -0.01 & 0.53 & 0.55 \\
\hline
\end{tabular}

\section{Interpretation}

1. The company's total income for the year 2015 was 124.80 , i.e.,61267.27, and for the year 2016 was $129.05 \%$, i.e., 61267.27 , and for 2017 was $136.02 \%$, i.e.,, 73660.76. In the year 2018, it is reduced to $131.69 \%$, i.e., 72385.52 , and the following year 2019it comes up to $122.89 \%$, i.e., 77912.35, which means that the income was increasing for the company.

2. The company's total expenses for the year 2015 were 24033.15 , i.e., 3.47, and 2016 were $44.28 \%$, i.e., 15319.89 but in the year 2017 , the costs of the company reduced to $49.89 \%$, i.e., 27019.91. And for the year 2018, the company's expenses again came up to $24337.24 \%$, i.e., 29.05 and in the year 2019 , the costs came up to $49.96 \%$, i.e., 2197.42 which means the prices of the company were increasing. This shows that the company is not working correctly.

\section{FINDINGS}

- The Total Assets of ICICI Bank have increased from 2015-2019.

- The current assets have increased during the period 20015-2019. 
- The current liabilities also have increased during the period 2015-2019.

- Profit after tax for the year ended March 312019 (FY2019) was Rs.3400 Cr., compared to Rs.6800 Cr. for the year ended March 312018 (FY2018).

- Net Interest Income increased from Rs. 40,400 Cr. in FY2018 to Rs. 41500 Cr. in FY 2019. It Increases due to improvement in net interest margin.

- Operating Expenses increased to Rs. 18100 Cr. in FY 2019 from Rs. 15700 Cr. in FY 2018. Asset Turnover Ratio remained constant at 0.6 from FY 2015-2017.

- The deposits trend was 117 in the FY 2015- 16, but it has been constant in FY 20162017 and FY 2017-18.

- Advances have drastically fallen to 104 in FY 2016-2017 from 113 in FY 2015-2016.

- The earnings per share have decreased from 18.48in FY 2015 to -3.34 (negative) in FY 2016.

- However, it has increased to 16.98 IN FY 2017 and has again decreased to 8.7 in FY 2018.

- The debt-to-equity ratio remained highest in FY 2014-2015 and has decreased since 2015-2016. It has almost remained constant from FY 2015-2016 to FY 2018-2019.

- The total asset to debt ratio was highest in FY 2016-2017.

\section{CONCLUSIONS}

Based on the various strategies used in the financial analysis of ICICI Bank, we can conclude that the bank's financial position and performance are all satisfactory. The bank's revenue has gone up in value. The bank has succeeded in maintaining a cheap profit situation.

The bank continued to expand its branch network in India. India's banking network has increased from 4050 branches as of March 312015 to 5275 branches as of June 30 2019. The Bank's ATM network has risen from 12,091 ATMs from March 312015 to 15,589 ATMs by June 302019.

The bank has succeeded in expanding its budget, which has grown by almost $50 \%$ over the past five years. People are significant shareholders. The bank's most considerable success has been the dramatic increase in its deposits, which have always been its primary goal. Fixed and current currencies have shown an increasing trend.

Equity shareholders also enjoy a growing trend within profits in their capital. Although current assets and liabilities (current debt) is not, the satisfactory bank has succeeded in maintaining a stable solvency status over the years. Given that the ratio of foreign and internal shares does not matter, it is clear that the bank was using more external equity within the type of loan and loan rather than the owner's money. The bank's investment also shows an increase. As a result of the rise in development, bank interest from that development proves to be a significant source of income for the bank.

A pioneer in digital banking transformation in India, ICICI Bank has been at the forefront of developing solutions, making banking more straightforward and more accessible for its customers. In line with its "Khayaal Aapka" philosophy, the bank offers digital solutions made up of specific episodes. With solutions that make banking easily accessible, convenient, and time-consuming, ICICI Bank continues to work with the state to empower its citizens digitally. 


\section{SUGGESTIONS}

- The bank's profit in the duration of the course is unsatisfactory. Yields are increasing but not at the same pace as costs due to the high reliance on credit in borrowing and borrowing capital.

- To improve profitability, the bank should reduce its dependence on foreign stocks to meet financial needs. As a result, interest costs will decrease, and profits will increase due to the bank. Similarly, non-productive costs should be reduced to maximize profits.

- Though the bank has successfully increased its deposits through new and attractive schemes, it should provide loans to needy persons with lower interest rates and a shorter maturity period for FD's.

- To achieve rural development's objective, it should open many branches in different rural areas of the country. It will facilitate in providing help to poor rural farmers and other living below the poverty line.

- Training programs should be devised for all staff, including call center and Staff of Direct Sales Associates or Associates of these banks. More importance should be given to upgrading product knowledge.

- Banks should try to finance more and more projects. Financing will help it to earn a higher number of profits.

- It should improve its day-to-day services to its customers. Such assistance can be enhanced by providing prompt services and demonstrating a cooperative attitude towards their customers. It will help to give a sense of community confidence and build a better public image.

- To increase the profit of the bank, the bank should decrease its operating expenses.

- To increase its liquidity bank should keep some more cash in its hands instead of giving more and more advances.

- The bank should manage all risks such as credit, market \& Operational risk correctly and be managed by a highly skilled and qualified person.

- The bank should adopt customer-oriented service delivery.

\section{LIMITATIONS}

- Difficulty in data collection.

- Limited knowledge about the bank in the initial stages.

- The analysis and interpretation are based on secondary data

- Contained in the published annual reports of ICICI Bank for the

- Study period.

- The ratio itself will not completely show the company's good or bad

- Financial position.

- The study of financial performance can be only a means to know About the financial condition of the company and cannot show aThrough picture of the activities of the company. 


\section{REFERENCES}

[1] Accountancy. R.K. Mittal, A.K. Jain.

[2] Financial Management- Theory and Practice. Shashi. K. Gupta, R.K. Sharma.

[3] Analysis of Financial Statement - T.S. Grewal Sultan Chand

[4] www.Icicibank.Com

[5] www.Moneycontrol.Com

[6] www.Money.Rediff.Com

[7] www.Wikipedia.Org

[8] www.Google.Com

[9] www.Scribd.Com

[10] www.idocpub.com

[11] www.business-standard.com

[12] www.standardmedia.co.ke

[13] www.coursehero.com

[14] www.platinumessays.com

[15] www.ukessays.com

[16] finpedia.co 